\title{
Vibration-based SHM for cultural heritage preservation: the case of the S. Pietro bell-tower in Perugia
}

\author{
Gabriele Comanducci ${ }^{1, a}$, Nicola Cavalagli ${ }^{1}$ and Filippo Ubertini ${ }^{1}$ \\ ${ }^{1}$ University of Perugia, Department of Civil and Environmental Engineering, 06125 Perugia, Italy
}

\begin{abstract}
In the present work, multivariate statistical analysis techniques are newly applied in the field of condition assessment of cultural heritage structures. More specifically, the paper presents the design and the implementation of an SHM system for the bell-tower of the Basilica of San Pietro, one of the most relevant monuments of the city of Perugia, Italy. The system comprises three high-sensitivity accelerometers permanently installed on top of the tower and a remote server that automatically processes the data so as to acquire modal parameters and to use such information for novelty analysis and health assessment. In the paper, after a brief description of the permanent monitoring system installed on the structure and of the adopted SHM strategy, the results of the first months of continuous monitoring are presented. Later on, the potential capability of the aforementioned statistical techniques in damage detection is verified by using the continuously identified eigenfrequencies of the bell tower.
\end{abstract}

\section{Introduction}

Ambient Vibration Testing (AVT) and Operational Modal Analysis (OMA) are widespread and reliable methods of modal testing in civil engineering. While they are commonly carried out in flexible structures, such as bridges and cables, in recent years a particular attention has been also devoted to their application for the conservation of monumental buildings. In this regards, vibration-based structural health monitoring (SHM) systems have revealed in many applications their capability of enabling automated condition assessment of slender structures with a limited number of sensors [1-6], leading to a cost-effective optimization of maintenance activities. However, the development of SHM systems able to early detect and alert about the occurrence of structural anomalies still remains a challenge in many cases. Methods of multivariate statistical analysis, such as principal component analysis and novelty detection, may overcome this issue, and even if documented validations of their effectiveness at damage detection in full-scale structures are not yet available, their application, especially in bridge engineering, is becoming very popular.

Equipping historical and monumental constructions with permanent SHM systems may lead to an optimal employment of the economic resources available for maintenance and rehabilitation activities, especially after seismic events. In fact, permanent SHM systems possess all the advantages of both AVT and OMA, being fully non-destructive and minimizing the interferences with the normal use of the structure but also allowing a continuous tracking of the actual condition of the structure, typically using a limited number of sensors. For these reasons, applications of vibration-based diagnostic and monitoring techniques to historic monumental buildings are becoming popular [7-12].

The authors have recently started a research project for monitoring of two relevant historical constructions in Italy: the bell-tower of the Basilica of San Pietro in Perugia and the dome of the Basilica of Santa Maria degli Angeli in Assisi. This paper presents the vibration-based monitoring system of the bell-tower of the Basilica of San Pietro. An innovative technique, combining automated mode tracking, multiple data regression, principal component analysis and novelty detection is proposed for automatically revealing any anomaly in the structural behaviour. The results of about five months of monitoring demonstrate the ability of the system in revealing even small changes in the structural behaviour, possibly related to a developing damage pattern, and show a promise towards a more widespread and systematic implementation of vibration-based SHM systems for cultural heritage preservation.

\section{The San Pietro bell-tower}

The Basilica of San Pietro in Perugia is located in the southern part of the City. The abbey was erected in 996 while the first erection of the bell-tower (Figure 1) dates back to the 13 th century.

Throughout the centuries the bell-tower was subjected to several structural and architectural interventions. The actual configuration dates back to the 15 th century and the design is attributed to the architect Bernardo Rossellino.

\footnotetext{
$\bar{a}$ Corresponding author: comanducci@strutture.unipg.it
} 


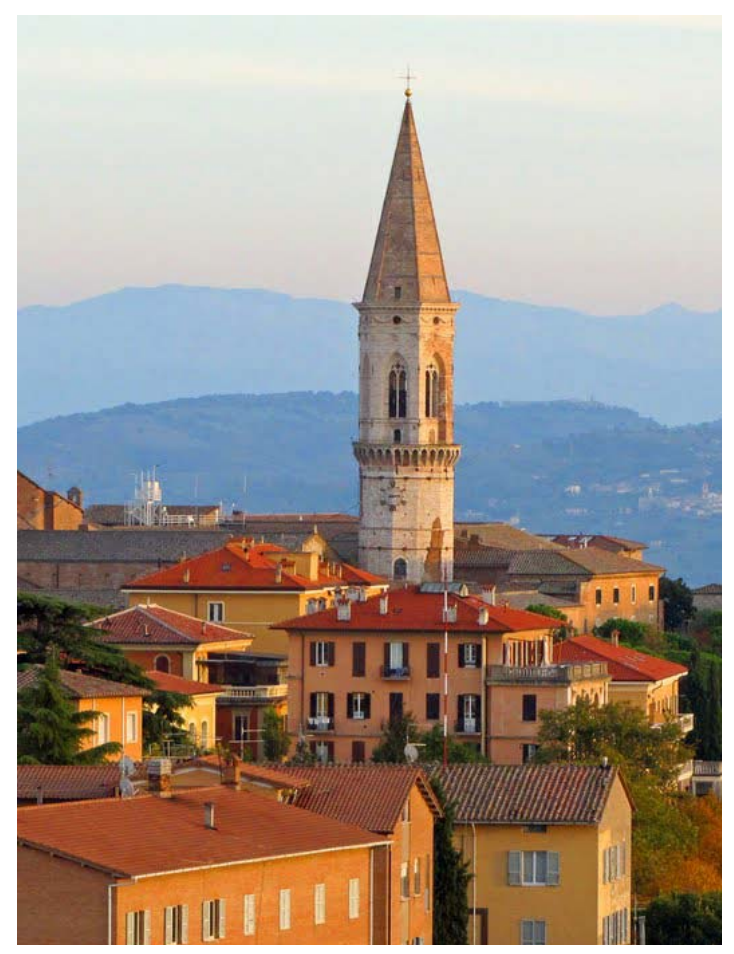

Figure 1. The San Pietro Bell-Tower.

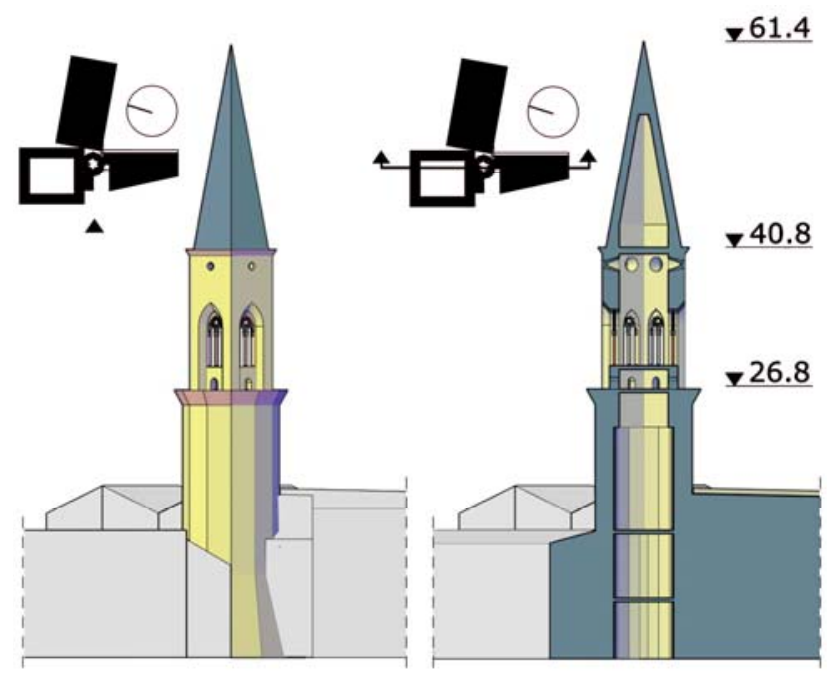

Figure 2. Solid CAD model of the bell-tower.

Various structural interventions were necessary to repair damages caused by lightning shocks that several times threatened the stability of the structure.

In the last years, the restoration and consolidation measures for the damages occurred after the strong Umbria-Marche earthquake of 1997 were completed.

The Benedictine abbey consists of several architectural volumes, including the basilica, the convent and today other local institutions, arranged around three main cloisters. In this context, the bell tower stands out between the basilica and other branches of the abbey, with a total height of about $61.4 \mathrm{~m}$. In the first $17 \mathrm{~m}$ the structure is restrained by the bordering buildings, so that the tower is free to move only in the last $45 \mathrm{~m}$ (Figure 2).
The bell tower is constituted by a dodecagonal shaft in the first $26 \mathrm{~m}$, a belfry with hexagonal cross section reaching an height of about $41 \mathrm{~m}$ and a cusp at the top. The constituent material is not homogeneous.

The shaft is made of stone masonry, with large external portions realized in brick masonry as structural rehabilitation measures due to the occurrence of several damages. The belfry and the cusp are made of brick masonry, but the former is characterized by an external curtain of stones. Moreover the belfry exhibits high mullioned windows in each of the six sides, thus resulting in a significant slenderness degree in the upper part of the structure.

\section{Monitoring system and data analysis}

\subsection{AVT and modal characterization}

AVT and OMA of the bell-tower were performed on February 16th 2015 when a fairly strong wind was blowing. The AVT was carried out by using high sensitivity accelerometers located in two sections of the bell-tower (Figure 3): at the base of the cusp (40.8 m) and at the base of the belfry $(29.1 \mathrm{~m})$. Uni-axial accelerometers model PCB 393B12 (10 V/g sensitivity) were used and data were recorded by using a 24-channel system, carrier model cDAQ-9188 with NI 9234 data acquisition modules (24-bit resolution, $102 \mathrm{~dB}$ dynamic range and anti-aliasing filters). The data were downsampled at $100 \mathrm{~Hz}$ for storage purpose.

Modal parameters of the bell-tower were extracted from AVT data by using 30-minutes long time histories and by application of a fully automated Stochastic Subspace Identification (SSI) technique [13].

Table 1 summarizes the values of identified natural frequencies and corresponding modal damping ratios, where mode types are referred to the reference axes depicted in Figure 3. Identified mode shapes are shown in Figure 4.

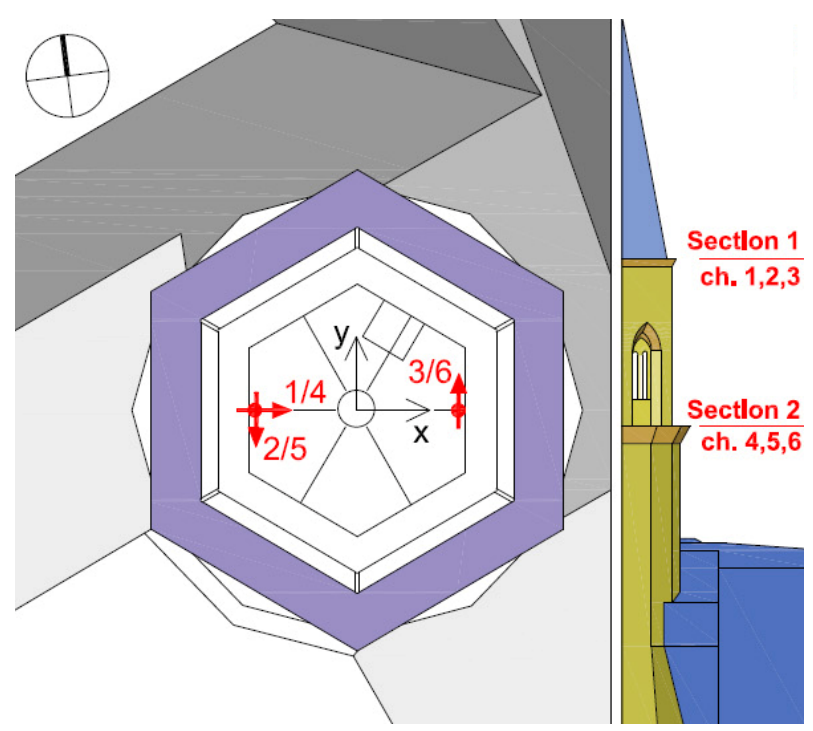

Figure 3. Sensors' layout in AVT. 
(a)

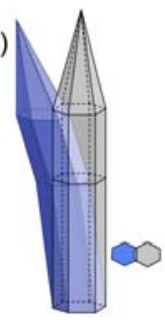

(b)
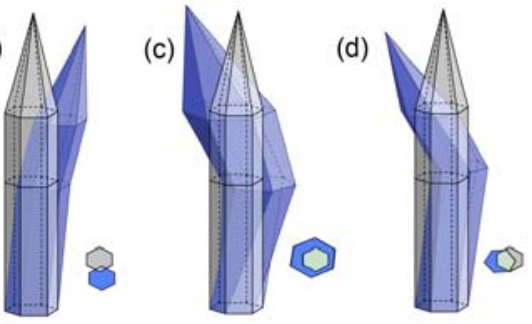

(e)

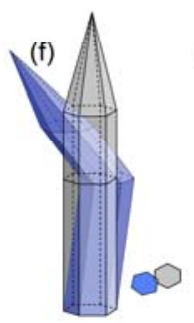

(g)

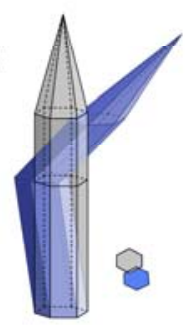

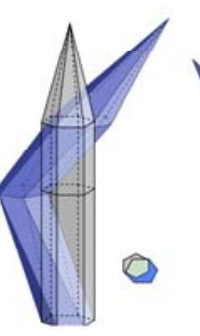

Figure 4. Identified mode shapes of the bell-tower.

Table 1. Identified modal frequencies and damping ratios of the bell-tower.

\begin{tabular}{|c|c|c|c|}
\hline Mode & $\begin{array}{c}\text { Frequency } \\
(\mathbf{H z})\end{array}$ & $\begin{array}{c}\text { Damping } \\
\text { ratio (\%) }\end{array}$ & Type \\
\hline 1 & 1.449 & 1.0 & $\mathrm{Fx} 1$ \\
\hline 2 & 1.518 & 1.0 & $\mathrm{Fy} 1$ \\
\hline 3 & 4.342 & 1.6 & $\mathrm{~T} 1$ \\
\hline 4 & 4.575 & 1.1 & $\mathrm{Fx} 2$ \\
\hline 5 & 4.889 & 2.3 & $\mathrm{Fy} 2$ \\
\hline 6 & 7.245 & 5.1 & $\mathrm{Fx} 3$ \\
\hline 7 & 7.263 & 2.7 & $\mathrm{Fy} 3$ \\
\hline
\end{tabular}

\subsection{Monitoring system}

The continuous monitoring system comprises three high sensitivity uni-axial piezoelectric accelerometers $(10 \mathrm{~V} / \mathrm{g}$ sensitivity) installed at the base of the cusp, with the configuration depicted in Figure 5.

The continuous monitoring data are recorded using the same data acquisition system used in the AVT, connected to a host PC located on site (Figure 6). Data are recorded at about $1600 \mathrm{~Hz}$, down-sampled at $100 \mathrm{~Hz}$ and stored in separate files of 30 recording minutes. The recorded data are then sent through the internet to a remote server located in the Laboratory of Structural Dynamics of the Department of Civil and Environmental Engineering of University of Perugia, where they are processed through an had-oc developed MatLab code. Figure 7 shows the location of the bell-tower and its network connection to the laboratory. Figure 8 shows remote access for quality control of the acquired data during monitoring.

The data processing code comprises the following steps:

- pre-processing analysis for detecting and correcting spikes and other anomalies in the data.
- Identification and removal of acceleration data under the excitation of the swinging bells.

- Low-pass filtering and decimation of the data to $40 \mathrm{~Hz}$.

- Application of the fully automated SSI modal identification procedure.

- Modal tracking based on a similarity check between estimated modal parameters.

The dynamic monitoring started on December 9th 2014 and the data reported in this paper cover a continuous monitoring period lasting up to the end of March 2015. Within the same monitoring period, temperature and wind speed data recorded by a weather station located nearby the bell-tower are also available. As better explained in the following developments of the paper, these data have allowed to perform preliminary investigations on the correlations between modal parameters of the bell tower and environmental conditions.

\subsection{Damage detection methodology}

Within the permanent monitoring system, any change in the dynamic behaviour of the bell-tower, possibly related to some developing damage pattern, is automatically detected by application of statistical process control tools to the time histories of identified modal frequencies.

The statistical process control tools adopted in the present study have a twofold purpose: (i) to remove the effects of changes in environmental and operational conditions from identified frequency time histories and (ii) to detect changes in the frequency data, in the form of statistical outliers, that arouse suspicion about the possible development of a structural damage.

In the present study, the bulk of data stemming from the permanent monitoring system is first processed through the afore-presented automated modal identification and modal tracking procedures. Then, the classical techniques of Multivariate Linear Regression (MLR) and Principal Component Analysis (PCA) are combined in a single statistical tool which is adopted for removing environmental effects. Finally, a technique of novelty analysis is adopted for damage detection. The adopted statistical process control tools are described below.

Tracked modal frequencies are collected in an $n \times N$ dimensional observation matrix, $\mathbf{Y}$, where $N$ is the number of observations and $n$ is the number of identified frequencies.

The residual error matrix, $\mathbf{E}$, is defined as follows for the purpose of damage detection:

$$
\mathbf{E}=\mathbf{Y}-\hat{\mathbf{Y}}
$$

where $\hat{\mathbf{Y}}$ are modal frequencies independently estimated through a proper statistical model able to reproduce the variance associated with changes in environmental parameters.

After computing matrix $\mathbf{E}$ in Eq. (1), a damage condition is identified as an anomaly in the residuals, under the assumption that damage induces a change in the distribution of E. To this aim, the classic statistical process control tool named Novelty Analysis is adopted. It 
basically consists of the use of control charts based on a properly defined statistical distance. In this application the $T^{2}$-statistic is exploited, which is defined as

$$
T^{2}=r(\overline{\mathbf{E}}-\overline{\overline{\mathbf{E}}})^{T} \boldsymbol{\Sigma}^{-1}(\overline{\mathbf{E}}-\overline{\overline{\mathbf{E}}})
$$

where $r$ is an integer parameter, referred to as group averaging size, $\overline{\mathbf{E}}$ is the mean of the residuals in the subgroup of the last $r$ observations, while $\overline{\overline{\mathbf{E}}}$ and $\boldsymbol{\Sigma}$ are the mean values and the covariance matrix of the residuals, respectively. Both quantities are statistically estimated in a reference period in which the structure is in the healthy state, which is called the training period.

An anomaly in the data is identified in the form of an outlier, that is a value of the statistical distance which lies outside fixed control limits. In the present application, the lower control limit is 0, while the Upper Control Limit (UCL) is statistically computed as the value of $T^{2}$ corresponding to a cumulative frequency of $95 \%$ in the training period. In this way, if data collected in the training period are statistically meaningful (the training period is sufficiently long), there is approximately a $5 \%$ probability to observe an outlier when the structure is in the healthy state (false alarm). Conversely, if a relative frequency of outliers significantly greater than the $5 \%$ is steadily observed over time, a change in the statistical distribution of the residuals is supposed to have occurred, so denoting an anomalous structural condition not experienced during the training period.

In the present application, the tools of MLR and PCA are combined to achieve an effective damage detection methodology. In particular, a MLR model is adopted at first and, then, PCA is applied to the residuals of MLR. Following this approach, the residual error matrix to be used in novelty analysis, Eq. (2), is computed as

$$
\mathbf{E}=\mathbf{Y}-\hat{\mathbf{T}}^{T} \hat{\mathbf{T}}\left(\mathbf{Y}-\boldsymbol{\beta}^{T} \mathbf{Z}^{T}\right)
$$

where $\boldsymbol{\beta}^{T} \mathbf{Z}^{T}$ represent the MLR estimate of $\mathbf{Y}$ (matrix $\mathbf{Z}$ contains the predictors while matrix $\beta$ contains the weight coefficients of the model). In Eq. (3), $\hat{\mathbf{T}}$ is the reduced loading matrix of the residuals of the MLR model that is computed by retaining only the first $l$ columns of matrix $\mathbf{U}$ in the following equation:

$$
\mathbf{T}=\mathbf{U}^{T}
$$

that, in turn, is computed by singular value decomposition (SVD) as

$$
\left(\mathbf{Y}-\boldsymbol{\beta}^{T} \mathbf{Z}^{T}\right)\left(\mathbf{Y}-\boldsymbol{\beta}^{T} \mathbf{Z}^{T}\right)^{T}=\mathbf{U S}^{2} \mathbf{U}^{T}
$$

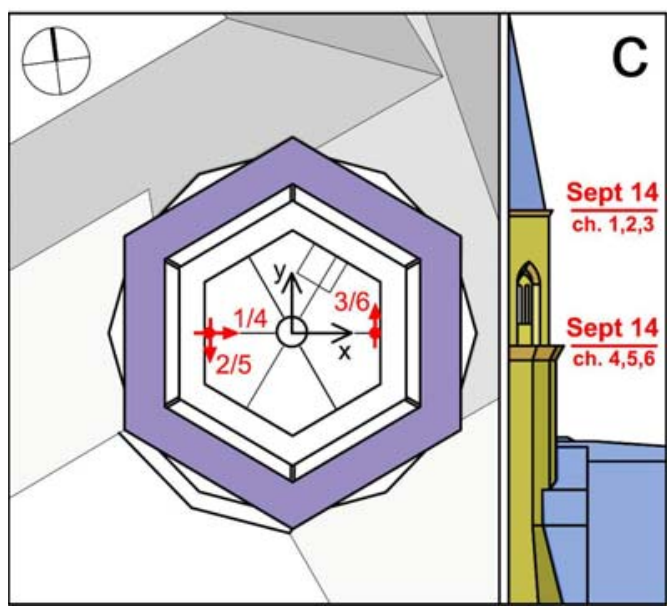

Figure 5. Sensors' layout of the monitoring system.

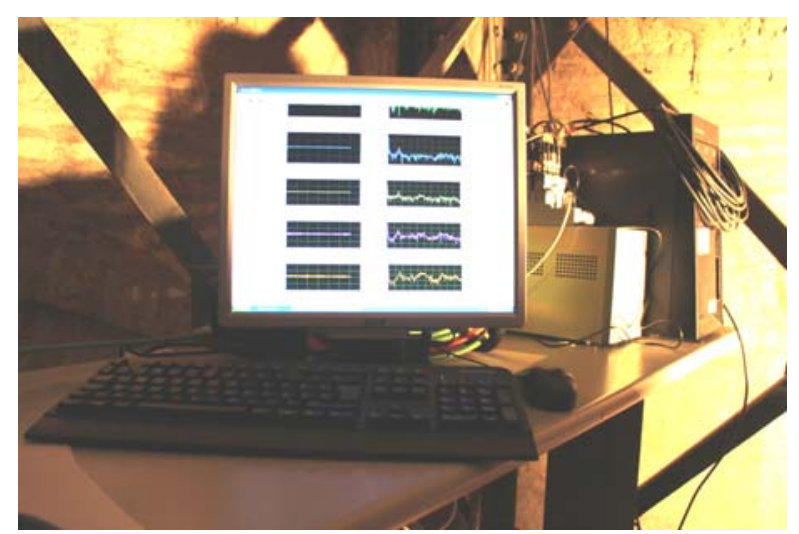

Figure 6. In-field data acquisition system.

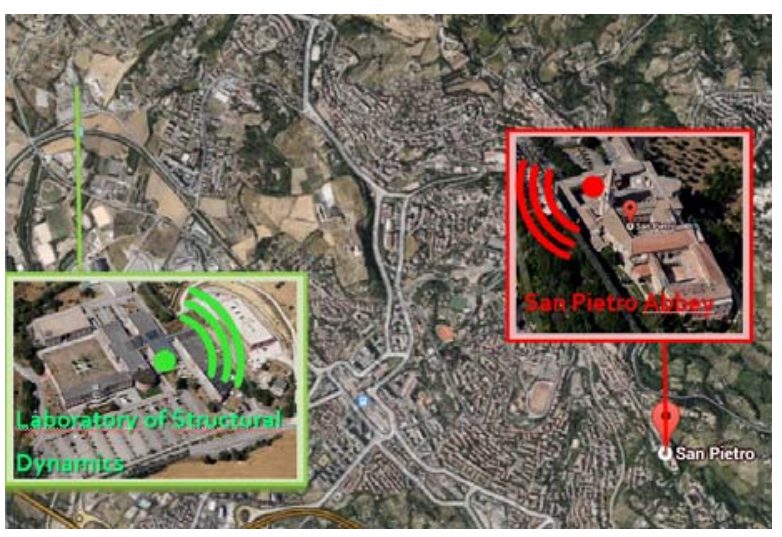

Figure 7. Remote data transmission.

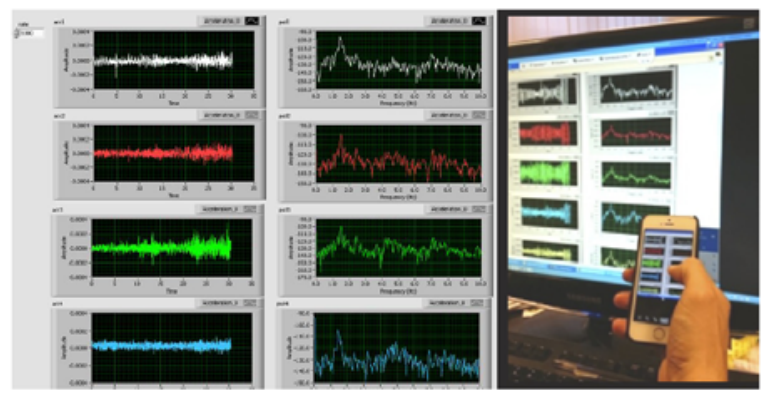

Figure 8. Remote access to monitoring data. 


\subsection{Analysis of monitoring data}

Figure 9 (a) shows time histories of identified natural frequencies of the bell-tower during about four months of monitoring. As shown from these results, the frequencies of all seven modes identified in the AVT are also consistently identified during monitoring, with the only exception of the fourth mode which is quite elusive, conceivably due to an insufficient level of excitation of this mode in operational conditions.

A detailed view of the time evolution of the first two eigenfrequencies, shown in the plot of Figure 9 (b), clearly highlights daily fluctuations of the natural frequencies, which are conceivably due to changes in environmental conditions, primarily air temperature. This has been checked by looking at correlations between natural frequencies and temperature of the air measured from the weather station located nearby the tower. The results, shown in Figure 10, clearly demonstrate frequency-temperature correlations, where frequencies are seen to increase with increasing temperature, because of micro-cracks closing in the masonry driven by thermal expansion. It should be noticed that correlation coefficients between frequencies and temperature could be higher than those observed in Figure 10, if temperature of masonry instead of air temperature would be considered.

Figure 11 shows the root mean square values of the accelerations recorded by the first sensor. The small levels of vibration are especially noteworthy, which is a consequence of the high stiffness of the structure which is slightly excited by wind. A little higher responses are observed when the excitation is provided by swinging bells, as shown in Figure 12.

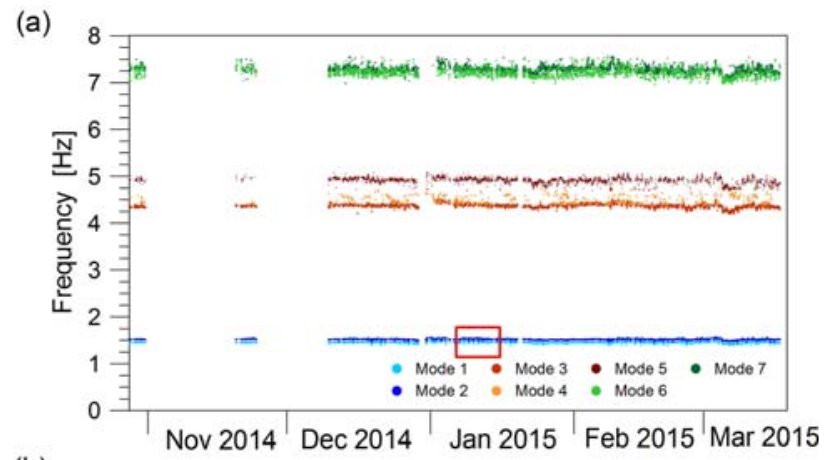

(b)

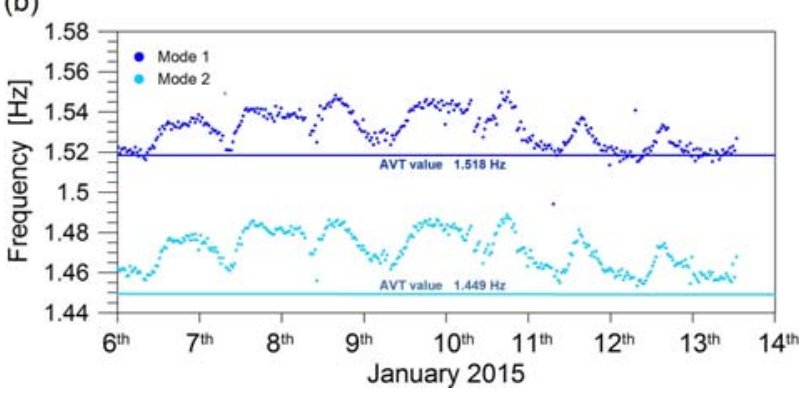

Figure 9. Time histories of the first seven modal frequencies identified from the permanent monitoring of the San Pietro bell tower (a) and detailed view highlighting daily fluctuations of the frequencies of the first two modes (b).
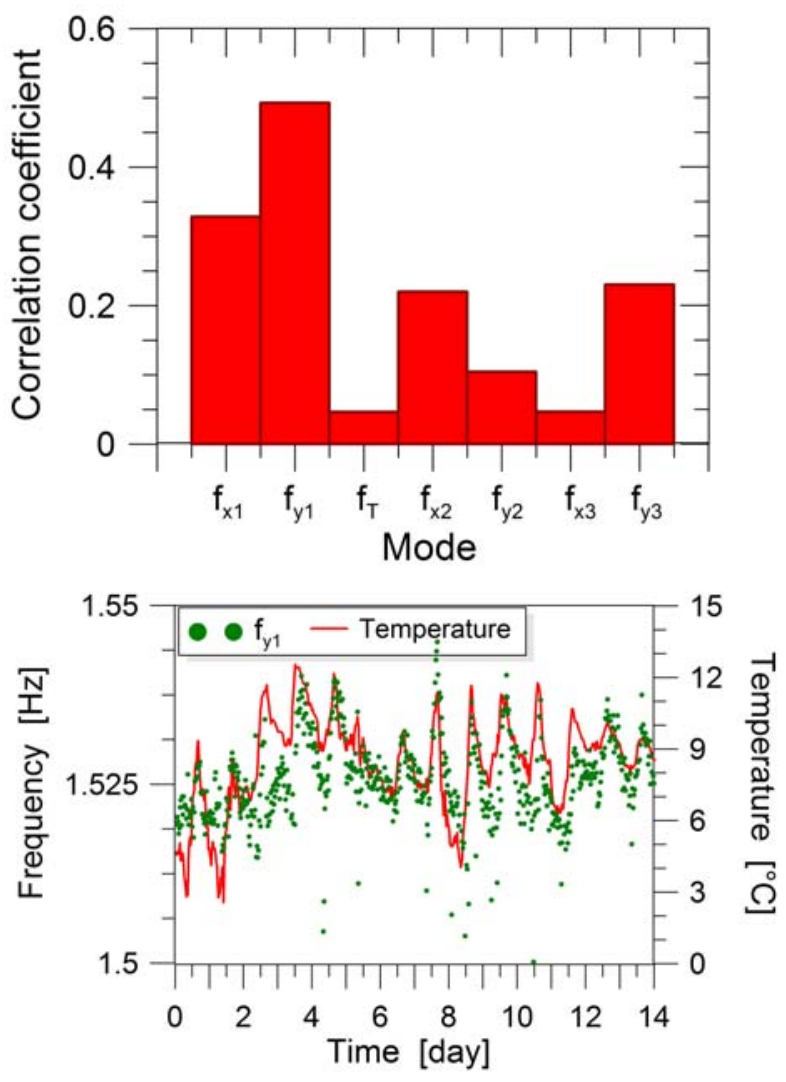

Figure 10. Correlation coefficients between identified frequencies and air temperature (top) and detailed view of the time histories of air temperature and first modal frequency (bottom)

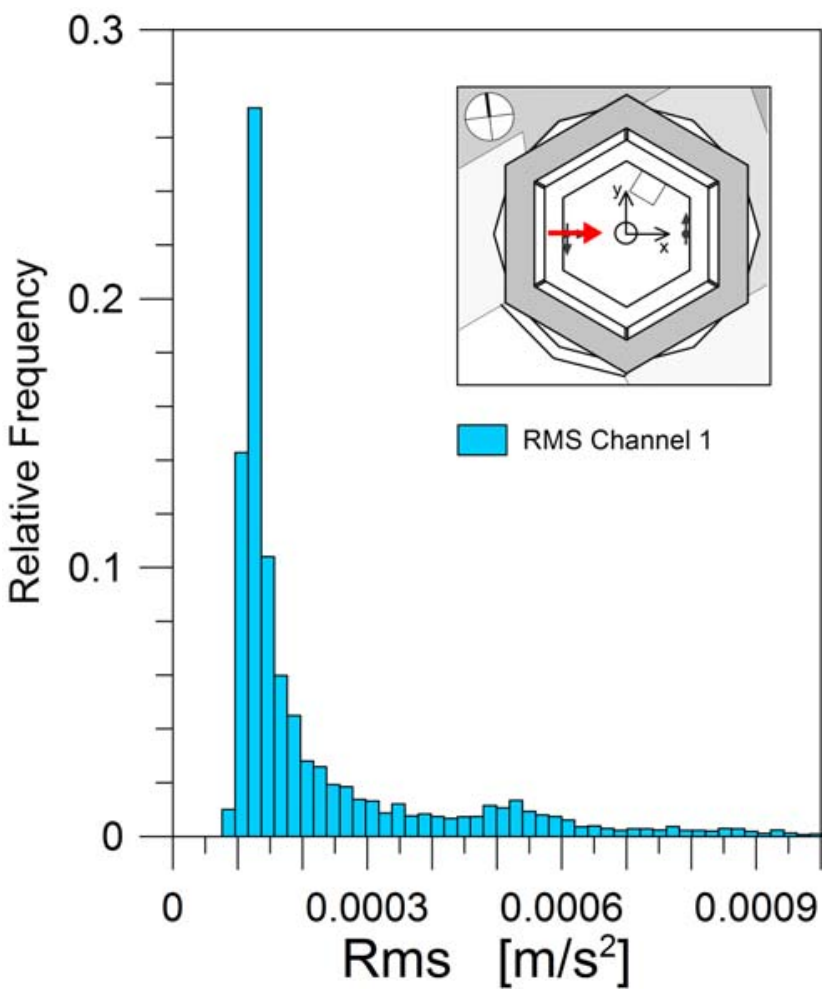

Figure 11. Histogram of root mean square (Rms) values of accelerations recorded by sensor number 1 during monitoring 


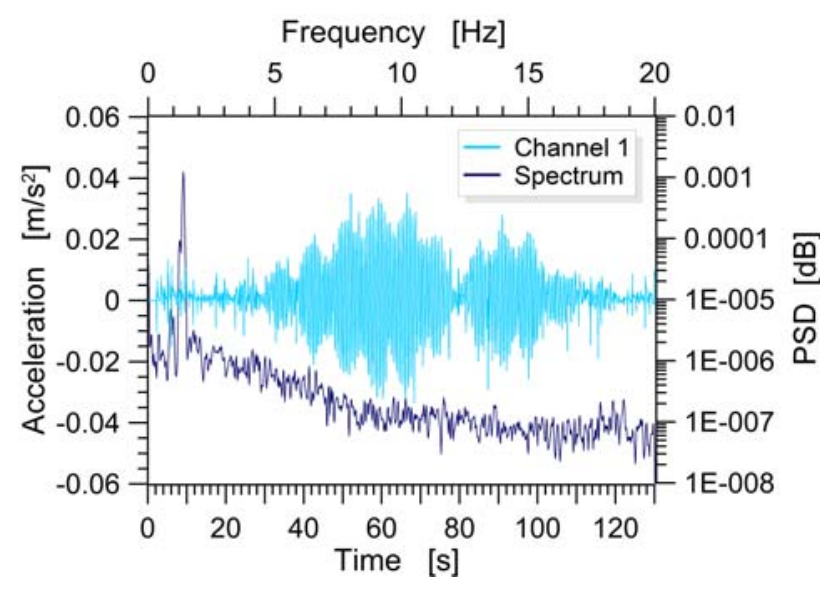

Figure 12. Dynamic response of the structure to a single swinging bell

\section{Numerical model and damage sensitivity}

With the purpose of investigating seismic vulnerability of the bell-tower, as well as frequency sensitivities to damage, a numerical finite element model of the structure has been constructed. The model, whose graphical sketch is depicted in Figure 13, is a linear elastic representation of the structure in its actual conditions, using threedimensional finite elements with orthotropic constitutive behaviour.

After some modal sensitivity analysis, not reported here for the sake of brevity, the numerical model has been manually tuned in order to match the experimentally identified modal properties. The main elastic properties of the materials constituting the tower, as estimated after such a tuning, are summarized in Table 2. In general, obtained results are very similar to what suggested in codes and in the literature for regular stone masonry (shaft) and for mixed stone-brick masonry in the remaining parts of the tower.

Table 3 summarizes identified and computed natural frequencies after manual tuning. These results highlight a good agreement between experimental results and numerical predictions, allowing to use the numerical model with some confidence. Mode shapes computed from the FE model are shown in Figure 14.

The tuned finite element model has been used to investigate sensitivity of natural frequencies to damage. In particular, a damage pattern similar to the one observed after field surveys following the strong seismic event of 1997 has been considered. It consists of damages at the base and the top of the columns of the belfry due to flexural failure of the columns themselves. This type of damage has been equivalently modelled as a localized reduction in stiffness. To this aim, a damage parameter has been introduced which represents the reduction in Young's modulus and in shear modulus imposed in the critical regions within the columns. Values of damage parameter have been variated from one column to another. In particular, one column is the most damaged one (maximum value of the damage parameter), while remaining columns are progressively less damaged by increasing the distance from the most damaged column. The column that is opposite to the most damaged one is considered to be in the healthy state.

The results of the damage sensitivity analysis of eigenfrequencies are shown in Figure 14. These results show that the most damage-sensitive frequency is the one of the third, torsional, mode. Progressively less sensitive are the frequencies of remaining modes.

Two damage scenarios are considered in particular. The first one, called D1, represents the damage condition producing a relative variation in the most sensitive frequency equal to $0.5 \%$, while the second one, called $\mathrm{D} 2$, corresponds to a $1.0 \%$ reduction in the frequency of the most sensitive mode. As shown in Figure 15, these damage conditions correspond to values of the damage parameter equal to 0.08 and 0.18 , respectively, corresponding to $8 \%$ and $18 \%$ localized reductions in stiffness in the critical regions of the most severely damaged column.
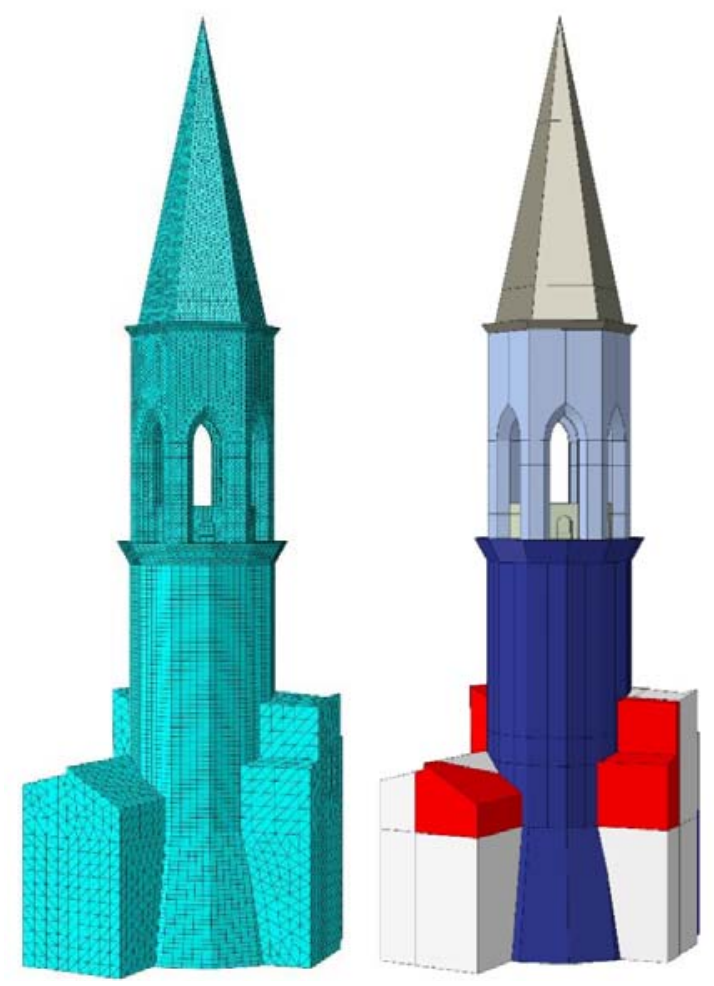

Figure 13. Finite element model (left) and topology of materials (right).

Table 2. Elastic parameters of the materials assumed in the numerical model

\begin{tabular}{|c|c|c|c|}
\hline Part & $\begin{array}{c}\text { Young's } \\
\text { modulus } \\
(\mathrm{MPa})\end{array}$ & $\begin{array}{c}\text { Shear } \\
\text { modulus } \\
(\mathrm{MPa})\end{array}$ & $\begin{array}{c}\text { Poisson's } \\
\text { ratio }\end{array}$ \\
\hline Shaft & 5450 & 1238 & 0.25 \\
\hline Belfry & 2950 & 1920 & 0.25 \\
\hline Cusp & 1500 & 500 & 0.25 \\
\hline
\end{tabular}




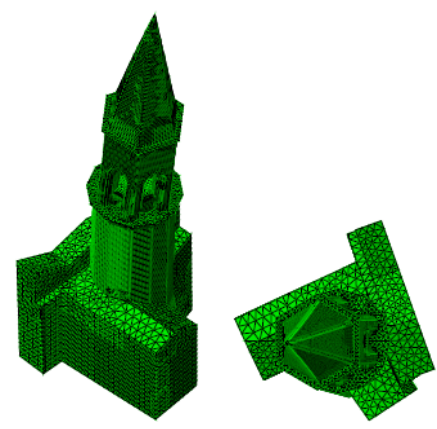

(a) $\mathrm{Fx} 1-1.448 \mathrm{~Hz}$
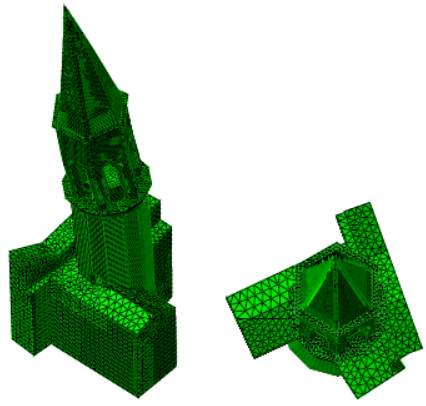

(b) Fyl $-1.518 \mathrm{~Hz}$

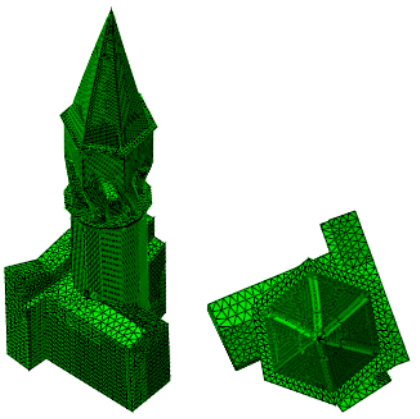

(c) $\mathrm{T} 1-4.356 \mathrm{~Hz}$

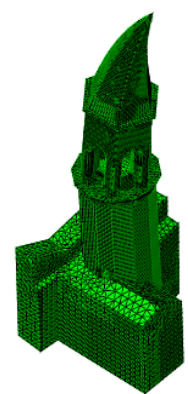

(d) $\mathrm{Fx} 2-4.577 \mathrm{~Hz}$

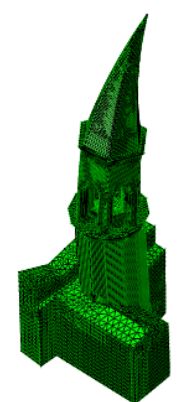

(e) Fy $2-4.692 \mathrm{~Hz}$
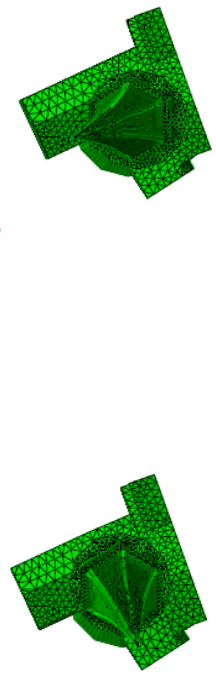

al (T) modal shapes obtained by the FE model

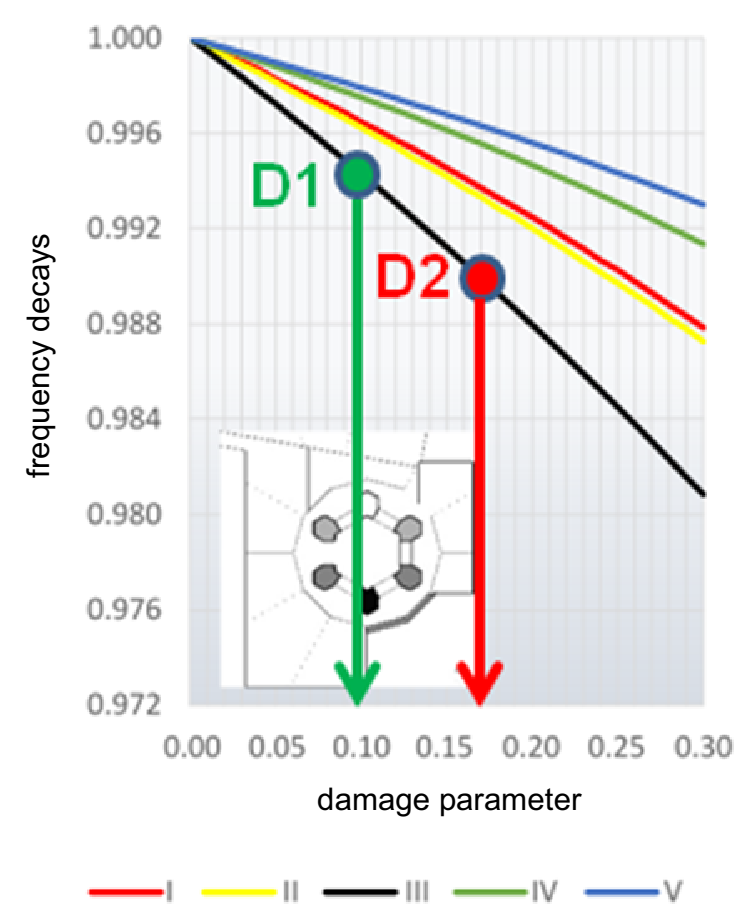

Figure 15. Decay of eigenfrequencies with damage parameter

Table 3. Comparison between identified and numerically predicted eigenfrequencies.

\begin{tabular}{|c|c|c|c|}
\hline Mode no & FEM (Hz) & ID (Hz) & $\Delta(\%)$ \\
\hline 1 & 1.448 & 1.449 & 0.07 \\
\hline 2 & 1.518 & 1.518 & 0.00 \\
\hline 3 & 4.356 & 4.345 & 0.25 \\
\hline 4 & 4.577 & 4.586 & 0.20 \\
\hline 5 & 4.692 & 4.861 & 3.49 \\
\hline
\end{tabular}

\section{Rapid post-earthquake assessment}

The damage detection methodology presented in Section 3.2 has been applied to monitoring data in order to test its ability in automatically revealing the presence of a damage in the structure. Damage patterns D1 and D2 are considered for this purpose (see Section 4) as typical damage conditions occurring after a relatively small earthquake.

Frequency shifts equal to those computed in Figure 15 have been artificially imposed to the time histories of the identified eigenfrequencies (Figure 9) starting from the $87^{\text {th }}$ day of monitoring. The frequencies identified in the first 80 days have been used for building the statistical models (MLR and PCA), as described in Section 3.3. The MLR model has been built by considering Rms amplitudes of the three measurement channels and the damping ratios of the first two modes as predictors, while three PCs have been retained in PCA. 
Figure 16 presents the control charts, in terms of the $T^{2}$ statistics, obtained for the two considered damage patterns. As shown in these plots, a significant increase in the number of outliers after the occurrence of the damage is observed in both D1 and D2 cases, where the percentage of outliers increases as expected with increasing damage severity. It is concluded, therefore, that the proposed SHM system enables rapid assessment of post-earthquake damages of the monitored bell-tower. It should be noticed that these results have been obtained with a training period of only 80 days, which does not allow to fully characterize fluctuations in natural frequencies of the bell-tower associated with seasonal changes in environmental conditions. It is expected, therefore, that increasing the number of available data sets will reduce the minimum level of damage that is detectable by the proposed SHM system.
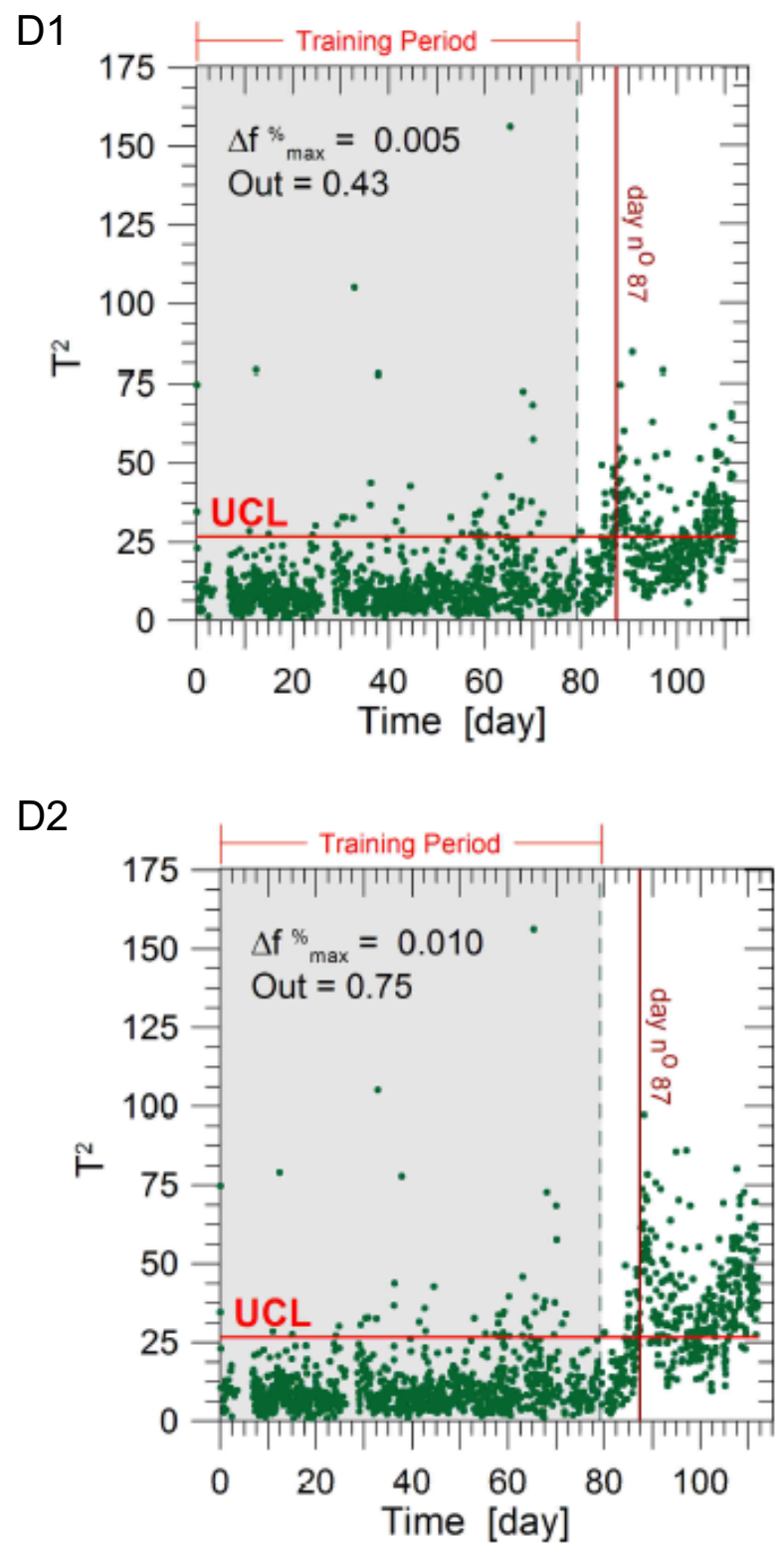

Figure 16. Control charts for automated damage detection (Out denotes the outliers' percentage after introducing the damage)

\section{Conclusions}

This paper has presented the development and implementation of a continuous vibration-based SHM system for rapid post-earthquake assessment of the monumental masonry bell-tower of San Pietro in Perugia, Italy. This monitoring system comprises three acceleration sensors whose records are remotely processed through an automated output only modal identification procedure and by a statistical process control tool that removes environmental effects and detects anomalies in identified eigenfrequencies enabling a prompt damage detection.

The analysis of the first four months of implementation of the system has clearly highlighted daily fluctuations of natural frequencies due to changes in temperature and, although with a still limited amount of data, has clearly demonstrated the ability of the proposed technique to allow damage detection.

\section{Acknowledgement}

The Authors gratefully acknowledge the financial support of the "Cassa di Risparmio di Perugia" Foundation that funded this study through the project "Structural Monitoring for the protection of the Cultural Heritage: the bell-tower of the Basilica of San Pietro in Perugia and the dome of the Basilica of Santa Maria degli Angeli in Assisi" (Project Code 2014.0266.021).

\section{References}

1. F. Magalhes, A. Cunha, E. Caetano, Mech Syst Signal Proc 28, 212-228 (2012)

2. A. Yan, G. Kerschen, P. De Boe, J. Golinval, Mech Syst Signal Proc 19, 847-864 (2005)

3. A. Yan, G. Kerschen, P. De Boe, J. Golinval, Mech Syst Signal Proc 19, 865-880 (2005)

4. A. Bellino, A. Fasana, L. Garibaldi, S. Marchesiello, Mech Syst Signal Proc 24, 2250-2260 (2010)

5. K. Worden, H. Sohn, C. Farrar, J Sound Vib 258, 741-761 (2002).

6. G. Comanducci, F. Ubertini, A. L. Materazzi, J Wind Eng Ind Aerodyn 141, 12-26 (2015).

7. A. Saisi, C. Gentile, M. Guidobaldi, Constr Build Mater 81, 101-112 (2015)

8. L. Ramos, L. Marques, P. Lourenco, G. DeRoeck, A. Campos-Costa, J. Roque, Mech Syst Signal Proc 24, 1291-1305 (2010).

9. S. Ivorra, F. J. Pallars, Eng Struct 28, 660-667 (2006).

10. C. Gentile, A. Saisi, Constr Build Mater 21, 13111321 (2007).

11. F. Pea, P. B. Lourenco, N. Mendes, D. V. Oliveira, Eng Struct 32, 1466-1478 (2010).

12. D. Foti, M. Diaferio, N. Giannoccaro, M. Mongelli, NDT E Int 47, 88-95 (2012).

13. F. Ubertini, C. Gentile, A. Materazzi, Eng Struct 46, 264-278 (2013) 